\title{
Treatment strategies for children with steroid-dependent nephrotic syndrome: in need of controlled studies
}

\author{
Uwe Querfeld $^{1}$ (D) $\cdot$ Lutz T. Weber $^{2}$
}

Received: 8 June 2018 / Accepted: 25 June 2018 / Published online: 2 July 2018

(C) IPNA 2018

Sir,

In their letter to the Editor [1], Fujinaga and Sakuraya briefly report details of their single-center experience with steroidsparing agents for the treatment of steroid-dependent nephrotic syndrome (SDNS) in children [2]. The variety of medications used [mycophenolate mofetil (MMF), cyclosporin A (CsA), cyclophosphamide, and mizoribine] reflects the dilemma of choosing the suitable long-term steroid-sparing agent in children with frequently relapsing nephrotic syndrome (FRNS) or SDNS that we have discussed in our review [3].

The authors have observed an association of MMF with reduced risk of relapse but increased risk of hospitalization compared to other immunosuppressants once the children had a febrile infection. This observation addresses the important questions of potential triggers activating relapse of the nephrotic syndrome and how to modify the trigger threshold. Since the original article is written in Japanese, we cannot comment on factors potentially contributing to febrile infections and relapse, but cumulative immunosuppression, length of treatment, combined therapy, sequential therapy, type of glomerulopathy, immunological and infectious parameters, and patient adherence might all play a role. Furthermore, the need for hospitalization and time of hospital stay are important criteria for treatment efficacy, and acute kidney injury is a frequent complication in children hospitalized with nephrotic syndrome [4].

The authors describe their center practice of using CsA prior to MMF as steroid-sparing agent in children with FRNS or SDNS and refer to our randomized prospective trial

Uwe Querfeld

Uwe.querfeld@charite.de

1 Pediatric Nephrology, Charité Campus Virchow, 13353 Berlin, Germany

2 University Children's Hospital, Cologne, Germany showing a higher efficacy of MMF after previous treatment with CsA [5], which we have discussed comprehensively in our review [3]. While we agree that sequential therapy with CsA and MMF may be an excellent option, the experience of Fujinaga and Sakuraya as well as our data indicates that more controlled prospective studies are needed to find better treatment strategies for FRNS and SDNS patients. We would completely agree that factors triggering relapse and/or febrile infections in children with FRNS/SDNS on maintenance immunosuppression should be among the topics in the focus of such future studies.

\section{Compliance with ethical standards}

Conflict of interest The authors declare that they have no conflict of interest.

\section{References}

1. Fuijnaga S, Sakuraya K (2018) Should mycophenolate mofetil be administered prior to cyclosporin A as a steroid-sparing agent to children with steroid-dependent nephrotic syndrome? Pediatr Nephrol. https://doi.org/10.1007/s00467-018-3993-4

2. Sakuraya K, Kakegawa D, Sakurai S, Hirano D, Fujinaga S (2018) Risk factors associated with relapse and hospitalization during periods of febrile infection in with nephrotic syndrome treated with immunosuppressant. [in Japanese] Nihon Shoni Jinzobyo Gakkai Shi 31(1):25-29

3. Querfeld U, Weber LT (2018) Mycophenolate mofetil for sustained remission in nephrotic syndrome. Pediatr Nephrol. https://doi.org/10. 1007/s00467-018-3970-y

4. Rheault MN, Zhang L, Selewski DT, Kallash M, Tran CL, Seamon M, Katsoufis C, Ashoor I, Hernandez J, Supe-Markovina K, D’Alessandri-Silva C, DeJesus-Gonzalez N, Vasylyeva TL, Formeck C, Woll C, Gbadegesin R, Geier P, Devarajan P, Carpenter SL, Kerlin BA, Smoyer WE, Midwest Pediatric Nephrology Consortium (2015) AKI in children hospitalized with nephrotic syndrome. Clin J Am Soc Nephrol 10:2110-2118

5. Gellermann J, Weber L, Pape L, Tonshoff B, Hoyer P, Querfeld U (2013) Mycophenolate mofetil versus cyclosporin A in children with frequently relapsing nephrotic syndrome. J Am Soc Nephrol 24: 1689-1697 